\title{
Open vs. laparoscopic surgery for locally advanced gastric cancer after neoadjuvant therapy: Short-term and long-term survival outcomes
}

\author{
NIANCHANG WANG ${ }^{1}$, AIPING ZHOU $^{2}$, JING JIN $^{3}$, HUANG HUANG $^{4}$, YAWEI ZHANG $^{4}$, \\ YINGTAI CHEN $^{1}$ and DONGBING ZHAO ${ }^{1}$ \\ Departments of ${ }^{1}$ Pancreatic and Gastric Surgical Oncology, ${ }^{2}$ Medical Oncology and \\ ${ }^{3}$ Radiation Oncology, National Cancer Center/National Clinical Research Center for Cancer/Cancer Hospital, \\ Chinese Academy of Medical Sciences and Peking Union Medical College, Beijing 100021, \\ P.R. China; ${ }^{4}$ Department of Surgery, Yale School of Medicine, New Haven, CT 06520, USA
}

Received July 11, 2018; Accepted June 12, 2019

DOI: $10.3892 / \mathrm{ol} .2020 .11626$

\begin{abstract}
The aim of the present study was to compare the short-term and long-term survival outcomes of laparoscopic gastrectomy vs. open gastrectomy in treating locally advanced gastric cancer (LAGC) after neoadjuvant therapy. This study retrospectively reviewed the medical records of 270 patients with LAGC, who underwent laparoscopic $(n=49)$ or conventional open $(n=221)$ surgery following neoadjuvant therapy between January 2007 and December 2016 in China National Cancer Center. Postoperative parameters and survival outcomes including overall survival and disease-free survival were analyzed. Patients who underwent laparoscopic gastrectomy (LP) had significantly shorter postoperative stay and a decreased number of metastatic lymph nodes harvested compared to those who underwent open surgery. The $75 \%$ disease-free survival (DFS) time in the laparoscopic surgery group (25.7 months) was higher compared with the open surgery group (15.6 months). However, no significant difference was observed in 5-year overall survival and DFS between the two groups. In conclusion, LG provides non-inferior shortand long-term survival outcomes compared with open surgery, suggesting a laparoscopic approach may be justified for patients with LAGC receiving neoadjuvant therapy. More randomized controlled trials are required to investigate the positive effects of LG for LAGC following neoadjuvant therapy.
\end{abstract}

Correspondence to: Dr Yingtai Chen or Dr Dongbing Zhao, Department of Pancreatic and Gastric Surgical Oncology, National Cancer Center/National Clinical Research Center for Cancer/Cancer Hospital, Chinese Academy of Medical Sciences and Peking Union Medical College, 17 Panjiayuan Nanli, Chaoyang, Beijing 100021, P.R. China

E-mail: yingtai.chen@hotmail.com

E-mail: dbzhao2003@sina.com

Key words: gastric cancer, laparoscopic surgery, neoadjuvant therapy, short-term outcomes, long-term survival outcomes

\section{Introduction}

Gastric cancer is the third leading cause of cancer-associated mortality worldwide (1). Locally advanced gastric cancer (LAGC) is a major treatment challenge and accounts for $80 \%$ of total gastric cancer cases in China $(2,3)$. The current therapeutic strategy for LAGC is multidisciplinary with a surgical procedure as the core. Accumulating evidence has revealed that neoadjuvant therapy improves the efficacy of LAGC compared with surgery alone (4-7).

Since the 2014 version of the guidelines of the Japan Society for Endoscopic Surgery, distal gastrectomy by the laparoscopic approach was recommended for stage I gastric cancer (8). For advanced gastric cancer following neoadjuvant therapy, however, the safety and efficacy of laparoscopic approach following were unclear, as oncologic outcomes of currently ongoing randomized trials are unknown $(9,10)$. A number of surgeons are now actively applying laparoscopic gastrectomy (LG) to patients with LAGC receiving neoadjuvant therapy. To the best of our knowledge, a limited number of studies have reported the safety and efficacy of LG following neoadjuvant therapy, particularly in terms of long-time survival.

Therefore, the aim of the present study was to evaluate the postoperative safety and efficacy and the long-time survival of patients who had undergone LG compared with patients who had undergone open gastrectomy (OG) following neoadjuvant therapy.

\section{Patients and methods}

Patient selection. This study retrospectively reviewed the medical records of 270 patients with LAGC who underwent LG $(n=49)$ or conventional OG $(n=221)$ surgery following neoadjuvant therapy between January 2007 and December 2016 at the China National Cancer Center (Beijing, China). LAGC was defined as clinical stage II-III according to the eighth edition American Joint Committee on Cancer (AJCC)/Union for International Cancer Control (UICC) 
A

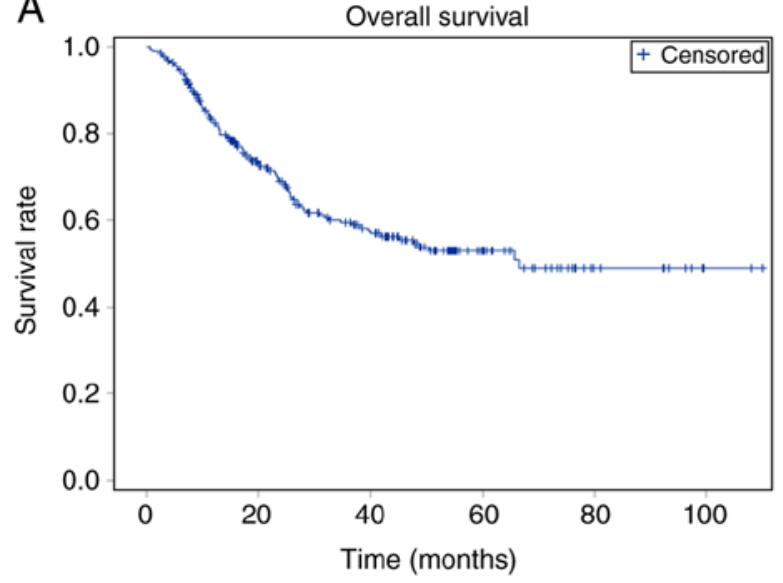

B

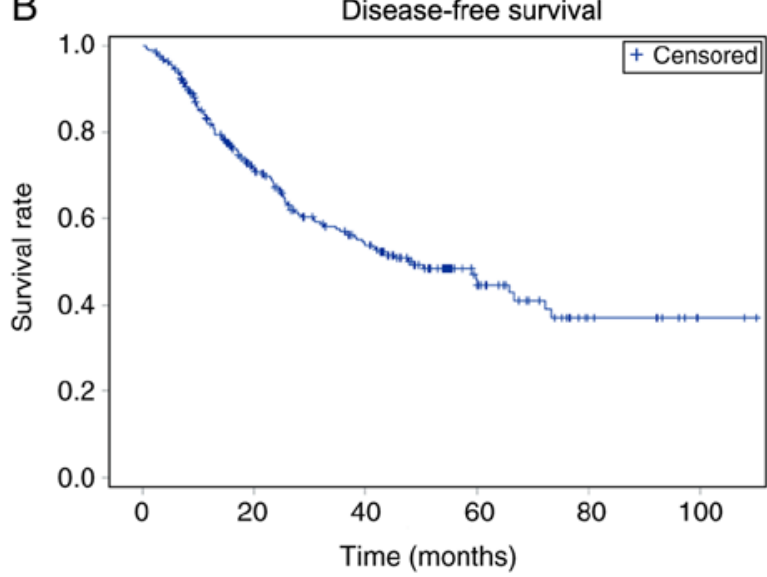

Figure 1. Overall survival and disease-free survival rates of patients who underwent surgery following neoadjuvant therapy.

Tumor-Node-Metastasis (TNM) gastric cancer classification (11). There were 188 male $(69.6 \%)$ and 82 female $(30.4 \%)$ patients (male-to-female ratio, 2.29:1; median age, 54.8 years; range, 28-84 years). The database included data on patient demographics, clinical history, past medical history, family history, comorbidities, diagnostic tests, tumor characteristics, therapeutic interventions, pathological data, postoperative parameters and survival outcomes. All data were backed up by source documents and the accuracy of the data was periodically reviewed. The study procedures were approved by the Institutional Review Board at the China National Cancer Center and the patients provided informed consent at the time of sample collection.

Procedures. Patients received a fluoropyrimidine-based chemoradiotherapy regimen preoperatively. Surgery was performed 2-8 weeks after completion of the neoadjuvant therapy. Histopathological examination was evaluated according to the Mandard Tumor Regression Grading evaluation system (12).

Follow-up. Patients were followed-up every 3 months for the first 2 years, every 6 months for the next 3 years, and every 6 months or yearly thereafter. For the postoperative follow-up, a physical examination, complete blood-cell count, liver function tests, serum carcinoembryonic antigen tests and chest radiography were performed every 3 months or 6 months; abdominal and pelvic computed tomography (CT) were performed every 6 months. Gastroscopic examinations were done 1 year postoperatively and once every 2 years thereafter. When a patient missed two consecutive scheduled visits or voluntarily withdrew consent to participate during the follow-up period, the patient was defined as lost to follow-up and their data were censored. The last follow-up was completed in May 2017 and included 251 patients.

Statistical analysis. Data of continuous variables are presented as the mean \pm standard deviation, whereas categorical variable data were presented as percentages. Patient demographic and clinical characteristics between the two groups were compared with Student's t-test for continuous variables with normal distribution and $\chi^{2}$ test for categorical variables. The Kaplan-Meier method was used to estimate disease-free survival (DFS) and overall survival (OS) rates, and the log-rank test was used to compare survival distribution. Multivariate Cox regression analysis was used to adjust for confounding factors that were significant in univariate analysis and for non-balanced between-group variables. Mean survival time (months) and 95\% confidence interval (CI) were calculated using the Kaplan-Meier method. A two-sided $\mathrm{P}<0.05$ was considered to indicate a statistically significant difference. All analyses were performed using SPSS statistical software version 24.0 (SPSS, Inc.).

\section{Results}

Between January 2007 and December 2016, 270 patients that underwent neoadjuvant therapy received LG $(n=49)$ or OG $(n=221)$. None of the patients had a metastatic lesion detected before or during the surgery. There were no deaths during the first 3 months after surgery. The OG and LG groups were balanced in terms of their baseline characteristics, combined comorbidities, clinical staging before neoadjuvant therapy, and chemotherapy regimens. The number of neoadjuvant therapy cycles was statistically different between the two groups $(\mathrm{P}=0.016)$. According to RECIST criteria (version 1.1) (13) and tumor regression grade, response on neoadjuvant therapy was not significantly different between the two groups (Table I).

Postoperative stay was shorter and the number of metastatic lymph nodes harvested was lower in the LG group compared with that in the OG group (Tables I and II). The number of resected lymph nodes was similar between the two groups (Table I). The incidence of complications, surgery time, blood loss and postoperative mortality were not significantly different between the two groups (Table II).

Fig. 1 shows the rates of DFS and OS of all patients, whereas Table III and Fig. 2 demonstrate the rates of DFS and OS in the LG and OG groups. The $75 \%$ DFS time was 15.6 (11.5-20.0) months for the OG group and 25.7 (12.3-41.3) months for the LG group. The 1-, 2-, 3- and 5-year OS rates for the LG group were $89.6,82.1,75.6$ and $65.8 \%$, respectively, and for the OG group were $81.6,65.9,55.9$ and $49.7 \%$, respectively. 
Table I. Demographic and clinicopathological characteristics in laparoscopy and open gastrectomy group.

\begin{tabular}{|c|c|c|c|}
\hline Variable & Laparoscopy group $(n=49)^{a}$ & Open gastrectomy group $(n=221)^{a}$ & P-value \\
\hline Age, years & $54.4(10.9)$ & $54.9(11.3)$ & 0.80 \\
\hline Body mass index, $\mathrm{kg} / \mathrm{m}^{2}$ & & & 0.89 \\
\hline$<18.5$ & $3(6.1)$ & $11(5.0)$ & \\
\hline $18.5-22.9$ & $22(44.9)$ & $91(41.2)$ & \\
\hline $23.0-27.4$ & $20(40.8)$ & $94(42.5)$ & \\
\hline$\geq 27.5$ & $4(8.2)$ & $25(11.3)$ & \\
\hline Sex, n $(\%)$ & & & 0.97 \\
\hline Male & $34(69.4)$ & $154(69.7)$ & \\
\hline Female & $15(30.6)$ & $67(30.3)$ & \\
\hline Comorbidities, n (\%) & & & 0.58 \\
\hline Diabetes & $3(6.1)$ & $16(7.2)$ & \\
\hline Coronary artery disease & $0(0.0)$ & $11(5.0)$ & \\
\hline Hypertension & $10(20.4)$ & $42(19.0)$ & \\
\hline Cerebral infraction & $0(0.0)$ & $5(2.3)$ & \\
\hline Hypothyroidism & $0(0.0)$ & $2(0.9)$ & \\
\hline Chronic obstructive pulmonary disease & $0(0.0)$ & $3(1.4)$ & \\
\hline Hepatitis B & $0(0.0)$ & $4(1.8)$ & \\
\hline Hepatitis C & $0(0.0)$ & $1(0.5)$ & \\
\hline Previous abdominal surgery, $\mathrm{n}(\%)$ & & & 0.78 \\
\hline Yes & $5(10.2)$ & $19(8.6)$ & \\
\hline No & $44(89.8)$ & $202(91.4)$ & \\
\hline Tumor location, n (\%) & & & 0.74 \\
\hline Upper $1 / 3$ & $13(26.5)$ & $54(24.4)$ & \\
\hline Middle 1/3 & $11(22.5)$ & $44(19.9)$ & \\
\hline Low $1 / 3$ & $9(18.4)$ & $61(27.6)$ & \\
\hline Upper-middle & $3(6.1)$ & $16(7.2)$ & \\
\hline Middle-low & $13(26.5)$ & $45(20.4)$ & \\
\hline Body-antrum & $0(0.0)$ & $1(0.5)$ & \\
\hline Family history of cancer, n (\%) & & & 0.47 \\
\hline Yes & $13(26.5)$ & $48(21.7)$ & \\
\hline No & $36(73.5)$ & $173(78.3)$ & \\
\hline Clinical TNM stage, $\mathrm{n}(\%)$ & & & 0.08 \\
\hline II & $4(8.2)$ & $5(2.3)$ & \\
\hline III & $45(91.8)$ & $216(95.5)$ & \\
\hline Neoadjuvant therapy regime, n (\%) & & & 0.10 \\
\hline XELOX & $3(6.1)$ & $11(5.0)$ & \\
\hline FOLFOX & $1(2.0)$ & $19(8.6)$ & \\
\hline SOX & $13(26.5)$ & $53(24.0)$ & \\
\hline SP & $2(4.1)$ & $19(8.6)$ & \\
\hline TXT+XELOX & $6(12.2)$ & $17(7.7)$ & \\
\hline $\mathrm{TCF}$ & $5(10.2)$ & $28(12.7)$ & \\
\hline DOS & $7(14.3)$ & $20(9.1)$ & \\
\hline $\mathrm{TXT}+\mathrm{SP}$ & $0(0.0)$ & $14(6.3)$ & \\
\hline Others & $5(10.2)$ & $28(12.7)$ & \\
\hline Cycle of neoadjuvant therapy, n (\%) & & & $0.02^{\mathrm{b}}$ \\
\hline $1-3$ & $23(46.9)$ & $87(39.4)$ & \\
\hline $4-6$ & $21(42.9)$ & $117(52.9)$ & \\
\hline$>6$ & $0(0.0)$ & $12(5.4)$ & \\
\hline
\end{tabular}


Table I. Continued.

\begin{tabular}{|c|c|c|c|}
\hline Variable & Laparoscopy group $(\mathrm{n}=49)^{\mathrm{a}}$ & $\begin{array}{l}\text { Open gastrectomy } \\
\text { group }(\mathrm{n}=221)^{\mathrm{a}}\end{array}$ & P-value \\
\hline Neoadjuvant therapy toxicity, $\mathrm{n}(\%)$ & & & 0.47 \\
\hline No toxicity & $13(26.5)$ & $74(33.5)$ & \\
\hline Grade I/II & $33(67.4)$ & $128(57.9)$ & \\
\hline Grade III/IV & $3(6.1)$ & $19(8.6)$ & \\
\hline Time between neoadjuvant therapy and surgery, days & $36.4(15.4)$ & $36.9(18.0)$ & 0.86 \\
\hline RECIST criteria (version 1.1), n (\%) & & & 0.93 \\
\hline Partial response & $34(69.4)$ & $150(67.9)$ & \\
\hline Stable disease & $13(26.5)$ & $64(29.0)$ & \\
\hline Progressive disease & $2(4.1)$ & $7(3.1)$ & \\
\hline Gastrectomy, n (\%) & & & 0.63 \\
\hline Distal & $29(59.2)$ & $117(52.9)$ & \\
\hline Proximal & $4(8.2)$ & $27(12.2)$ & \\
\hline Total & $16(32.7)$ & $77(34.8)$ & \\
\hline Borrmann type (22), n (\%) & & & 0.37 \\
\hline I & $4(8.2)$ & $8(3.6)$ & \\
\hline II & $18(36.7)$ & $62(28.1)$ & \\
\hline III & $23(46.9)$ & $123(55.7)$ & \\
\hline IV & $4(8.2)$ & $25(11.3)$ & \\
\hline Unknown & $0(0.0)$ & $3(1.4)$ & \\
\hline Lauren type (23), n (\%) & & & 0.35 \\
\hline Intestinal & $13(26.5)$ & $43(19.5)$ & \\
\hline Diffuse & $17(34.7)$ & $66(29.9)$ & \\
\hline Mixed & $8(16.3)$ & $34(15.4)$ & \\
\hline Unknown & $11(22.5)$ & $78(35.3)$ & \\
\hline Primary pathology, n (\%) & & & 0.65 \\
\hline Poorly differentiated adenocarcinoma & $32(65.3)$ & $146(66.1)$ & \\
\hline Moderately differentiated adenocarcinoma & $10(20.4)$ & $31(14.0)$ & \\
\hline Well differentiated adenocarcinoma & $1(2.0)$ & $3(1.4)$ & \\
\hline Signet ring cell carcinoma & $1(2.0)$ & $13(5.9)$ & \\
\hline Minor adenocarcinoma remains & $2(4.1)$ & $6(2.7)$ & \\
\hline No adenocarcinoma remains (complete response) & $2(4.1)$ & $18(8.1)$ & \\
\hline Other & $1(2.0)$ & $4(1.8)$ & \\
\hline Resected lymph nodes, $\mathrm{n}(\%)$ & $32.9(13.6)$ & $30.0(14.0)$ & 0.19 \\
\hline Metastatic lymph nodes, n (\%) & $2.4(3.4)$ & $6.0(9.1)$ & $<0.0001^{\mathrm{b}}$ \\
\hline Mandard Tumor Regression Grading (12), n (\%) & & & 0.06 \\
\hline 1 & $2(4.1)$ & $17(7.7)$ & \\
\hline 2 & $10(20.4)$ & $32(14.5)$ & \\
\hline 3 & $22(44.9)$ & $62(28.1)$ & \\
\hline 4 & $2(4.1)$ & $8(3.6)$ & \\
\hline 5 & $13(26.5)$ & $102(46.2)$ & \\
\hline
\end{tabular}

a Data are presented as the mean (standard deviation) for continuous variables and number (percentage) for categorical variables. ${ }^{\mathrm{b}} \mathrm{P}<0.05 . \mathrm{XELOX}$, oxaliplatin and capecitabine; FOLFOX, folinic acid, fluorouracil and oxaliplatin; SOX, S-1 and oxaliplatin; SP, S-1/cisplatin; TXT, docetaxel; TCF, docetaxel, carboplatin and 5-fluorouracil; DOS, docetaxel, oxaliplatin and S-1; RECIST, response evaluation criteria in solid tumors.

The 1-, 2-, 3- and 5-year DFS rates for LG group were 89.6, 79.8, 70.4 and 53.3\%, respectively, and for OG group were 81.1, $64.4,54.1$ and $43.7 \%$, respectively. No significant difference was observed in OS and DFS between the two groups. In addition, no significant difference was observed in 1-, 2-, 3- and 5-year DFS and OS. 
Table II. Comparison of perioperative parameters between the laparoscopic and open gastrectomy groups.

\begin{tabular}{|c|c|c|c|}
\hline Variable & Laparoscopy group $(n=49)^{a}$ & Open gastrectomy group $(\mathrm{n}=221)^{\mathrm{a}}$ & P-value \\
\hline Complication, n (\%) & $6(12.2)$ & $26(11.8)$ & 0.752 \\
\hline Central line infection & $0(0.0)$ & $1(0.5)$ & \\
\hline Wound infection & $0(0.0)$ & $3(1.4)$ & \\
\hline Renal failure & $0(0.0)$ & $1(0.5)$ & \\
\hline Multiple organ failure & $0(0.0)$ & $1(0.5)$ & \\
\hline Delayed gastric emptying & $1(2.0)$ & $1(0.5)$ & \\
\hline Gastrointestinal hemorrhage & $0(0.0)$ & $5(2.3)$ & \\
\hline Pleural effusion & $1(2.0)$ & $2(0.9)$ & \\
\hline Pneumonia & $0(0.0)$ & $3(1.4)$ & \\
\hline Fat liquefaction & $1(2.0)$ & $2(0.9)$ & \\
\hline Postoperative ileus & $0(0.0)$ & $3(1.4)$ & \\
\hline Intra-abdominal infection & $1(2.0)$ & $3(1.4)$ & \\
\hline Duodenal stump fistula & $1(2.0)$ & $1(0.5)$ & \\
\hline Anastomotic leak & $1(2.0)$ & $4(1.8)$ & \\
\hline Reoperation & $1(2.0)$ & $1(0.5)$ & \\
\hline Postoperative mortality & $0(0.0)$ & $0(0.0)$ & \\
\hline Surgery time, $\min$ & $221.5(69.9)$ & $201.1(56.7)$ & 0.060 \\
\hline Estimated blood loss, $\mathrm{ml}$ & $260.2(232.1)$ & $241.1(186.3)$ & 0.590 \\
\hline Time to pull gastric tube, days & $5.5(2.0)$ & $6.6(3.3)$ & $0.002^{\mathrm{b}}$ \\
\hline Postoperative stay, days & $11.1(4.4)$ & $13.0(7.3)$ & $0.020^{\mathrm{b}}$ \\
\hline
\end{tabular}

a Data are presented as the mean (standard deviation) for continuous variables and number (percentage) for categorical variables.

A

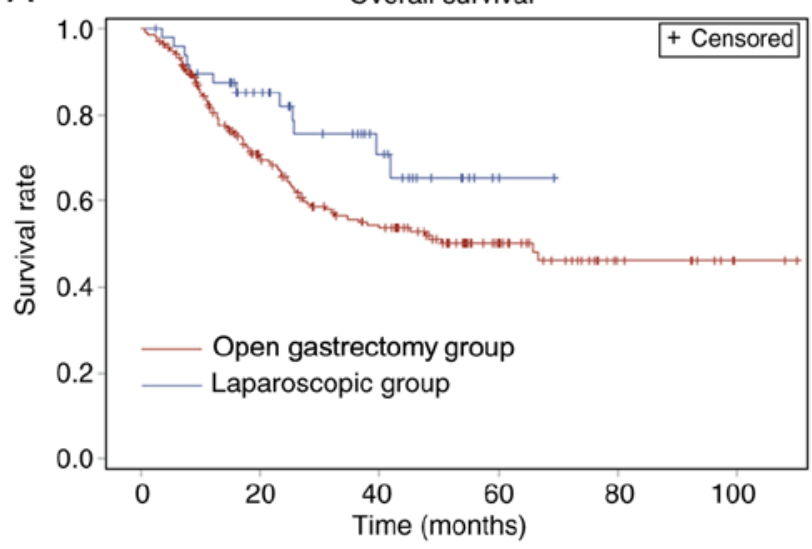

B

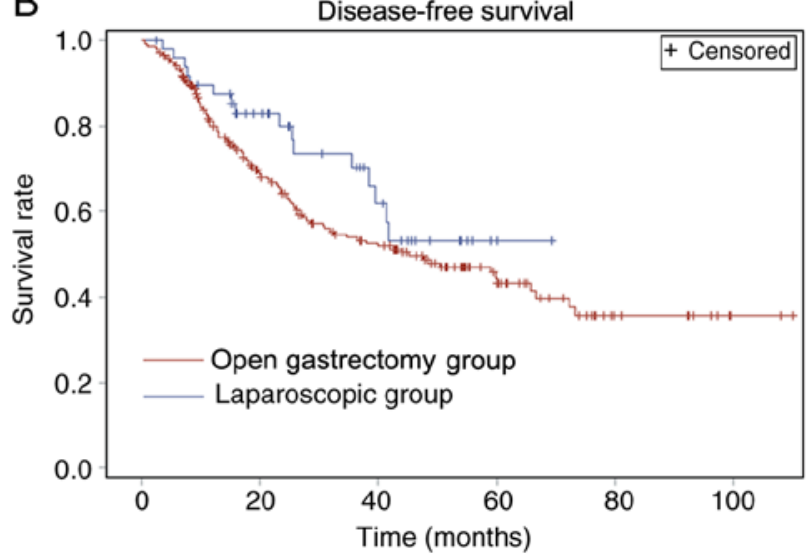

Figure 2. Comparison of the overall survival and disease-free survival rates between the open gastrectomy group and the laparoscopic group.

\section{Discussion}

According to the Clinical Practice Guidelines in Oncology Gastric Cancer (version 2.2018), patients with potentially resectable cT2 or higher, any $\mathrm{N}$, and cM0 tumors are recommended to receive perioperative chemotherapy (category 1) or perioperative chemoradiaton (category 2B) (14). Previous randomized control trials and retrospective studies have demonstrated the safety and efficacy of LG for LAGC (15-18). However, the evidence of safety and long-term results of laparoscopic surgery for the treatment of LAGC after neoadjuvant therapy were scarce.
A higher number of patients underwent conventional OG following neoadjuvant therapy in the China National Cancer Center compared with those that underwent LG, which may be due to the following reasons. First, $O G$ was selected for patients who were diagnosed with bulky lymph nodes or lymph nodes fused together by CT or MRI following neoadjuvant therapy. Second, a number of surgeons in the China National Cancer Center only perform OG. Third, several patients with severe coronary artery disease or pulmonary disease were assigned to the OG group for surgical safety.

The present study revealed that patients with LAGC that underwent LG after neoadjuvant therapy had significantly 
Table III. Comparison of survival status between laparoscopic and gastrectomy group.

\begin{tabular}{lccc}
\hline Variable & Laparoscopy group $(\mathrm{n}=49)$ & Open gastrectomy group $(\mathrm{n}=221)$ & P-value \\
\hline DFS, months $^{\mathrm{a}}$ & $25.7(12.3-41.3)$ & $15.6(11.5-20.0)$ & 0.27 \\
1-year rate & 0.896 & 0.811 \\
2-year rate & 0.798 & 0.644 \\
3-year rate & 0.704 & 0.541 \\
5-year rate & 0.533 & 0.437 & \\
OS $_{\text {months }}$ & $39.5(12.3-)^{\mathrm{b}}$ & $16.1(12.1-22.1)$ & 0.12 \\
1-year rate & 0.896 & 0.816 \\
2-year rate & 0.821 & 0.659 & \\
3-year rate & 0.756 & 0.559 & \\
5-year rate & 0.658 & 0.497 & \\
\hline
\end{tabular}

${ }^{2} 75 \%$ survival time; since survival rate at the longest time point exceeds $50 \%$ for the laparoscopic group, median survival time could not be computed. ${ }^{b}$ The upper confidence limit for the $75 \%$ OS time among the laparoscopic group could not be calculated due to the right-censoring of the data. OS and DFS times are presented as the mean (95\% confidence interval). DFS, disease-free survival; OS, overall survival.

shorter postoperative stay compared to OG (11.1 vs. 13.0; $\mathrm{P}=0.020$ ). A randomized controlled trial has confirmed that the benefits of the laparoscopic approach measured by early postoperative recovery can safely be offered to select patients with LAGC (19). The results from a prospective study also showed that laparoscopic distal gastrectomy after neoadjuvant therapy has comparable results with open distal gastrectomy in safety and efficacy in the short term (20).

The number of metastatic lymph nodes harvested in the LG group was less than that in the OG group (2.4 vs. 6.0; $\mathrm{P}<0.0001)$. Laparoscopic procedures have certain limitations, such as difficult management of tumors with bulky metastasis-positive nodes or large primary tumors, and unusual tissue fibrosis or edema may present following neoadjuvant therapy, which further increases surgical difficulty (21). Therefore, the majority of doctors select conventional OG.

Resected lymph nodes, incidence of complications, surgery time, blood loss and postoperative mortality were not significantly different between the two groups. The results were consistent with previous reports, which confirm the benefit of the laparoscopic approach, measured by early postoperative recovery, and that it can be safely offered to select patients with LAGC after neoadjuvant therapy $(14,15)$.

The OS and DFS of the laparoscopic group were indicated to be longer than the open gastrectomy group; however, there were no statistically significant differences between the two groups. The 75\% DFS time was 15.6 (11.5-20.0) months for the open surgery group and 25.7 (12.3-41.3) months for the laparoscopic surgery group $(\mathrm{P}=0.12)$. The upper confidence limit for the $75 \%$ OS time among the laparoscopic group could not be calculated due to the right-censoring of the data. The right-censoring may cause an underestimate of the mean survival time and its standard error. The major reason may be the small sample size; in addition, the follow-up time was short. The cycle of neoadjuvant therapy was statistically different between the two groups; however, it did not affect the long-term survival result of the study (data not shown).

Strengths and limitations should be considered when interpreting the study results. To the best of our knowledge, this cohort is the largest to date to compare the short-term and long-term survival outcomes between OG and LG for LAGC after neoadjuvant therapy. There were several limitations in this study. First, it is a retrospective study with a limited sample size in a single center. Second, patients were divided into different surgical approach groups, based on their personal choice, because either surgical type can be used according to doctors' clinical judgments. Furthermore, the follow-up time was short, and follow-up of these patients is ongoing.

The results of this study demonstrated that LG for LAGC following neoadjuvant therapy may provide non-inferior short-term and long-term survival outcomes compared with open surgery, suggesting a laparoscopic approach may be justified for patients with LAGC after neoadjuvant therapy. Multicenter randomized controlled trials are required to investigate the positive effects of LG for LAGC following neoadjuvant therapy.

\section{Acknowledgements}

Not applicable.

\section{Funding}

This research was supported by The National Key R\&D Program of China (grant. no. 2017YFC0908300).

\section{Availability of data and materials}

The datasets used and/or analyzed during the current study are available from the corresponding author on reasonable request.

\section{Authors' contributions}

YC, DZ, AZ and JJ conceived and designed the study. NW and $\mathrm{HH}$ collected the data. YC and YZ analyzed the data. NW wrote the manuscript. YC and DZ reviewed and revised the manuscript. All authors read and approved the final manuscript. 


\section{Ethics approval and consent to participate}

All study procedures were approved by the Institutional Review Board at the China National Cancer Center and the patients provided written informed consent at the time of sample collection.

\section{Patient consent for publication}

Not applicable.

\section{Competing interests}

The authors declare that they have no competing interests.

\section{References}

1. Ferlay J, Soerjomataram I, Dikshit R, Eser S, Mathers C, Rebelo M, Parkin DM, Forman D and Bray F: Cancer incidence and mortality worldwide: Sources, methods and major patterns in GLOBOCAN 2012. Int J Cancer 136: E359-E386, 2015.

2. Chen W, Zheng R, Baade PD, Zhang S, Zeng H, Bray F, Jemal A, Yu XQ and He J: Cancer statistics in China, 2015. CA Cancer J Clin 66: 115-132, 2016

3. Nashimoto A, Akazawa K, Isobe Y, Miyashiro I, Katai H, Kodera Y, Tsujitani S, Seto Y, Furukawa H, Oda I, et al: Gastric cancer treated in 2002 in Japan: 2009 annual report of the JGCA nationwide registry. Gastric Cancer 16: 1-27, 2013.

4. Cunningham D, Allum WH, Stenning SP, Thompson JN, Van de Velde CJ, Nicolson M, Scarffe JH, Lofts FJ, Falk SJ, Iveson TJ, et al: Perioperative chemotherapy versus surgery alone for resectable gastroesophageal cancer. N Engl J Med 355 $11-20,2006$

5. Yoshikawa T, Sasako M, Yamamoto S, Sano T, Imamura H, Fujitani K, Oshita H, Ito S, Kawashima Y and Fukushima N Phase II study of neoadjuvant chemotherapy and extended surgery for locally advanced gastric cancer. Br J Surg 96 . 1015-1022, 2009.

6. Ychou M, Boige V, Pignon JP, Conroy T, Bouché O, Lebreton G, Ducourtieux M, Bedenne L, Fabre JM, Saint-Aubert B, et al Perioperative chemotherapy compared with surgery alone for resectable gastroesophageal adenocarcinoma: An FNCLCC and FFCD multicenter phase III trial. J ClinOncol 29: 1715-1721, 2011.

7. Tsuburaya A, Mizusawa J, Tanaka Y, Fukushima N, Nashimoto A and Sasako M; Stomach Cancer Study Group of the Japan Clinical Oncology Group: Neoadjuvant chemotherapy with S-1 and cisplatin followed by D2 gastrectomy with para-aortic lymph node dissection for gastric cancer with extensive lymph node metastasis. Br J Surg 101: 653-660, 2014.

8. Japanese Gastric Cancer Association: Japanese gastric cancer treatment guidelines 2014 (ver. 4). Gastric Cancer 20: 1-19, 2017

9. Inaki N, Etoh T, Ohyama T, Uchiyama K, Katada N, Koeda K, Yoshida K, Takagane A, Kojima K, Sakuramoto S, et al: A Multi-institutional, prospective, phase II feasibility study of laparoscopy-assisted distal gastrectomy with D2 lymph node dissection for locally advanced gastric cancer (JLSSG0901) World J Surg 39: 2734-2741, 2015.
10. Hur H, Lee HY, Lee HJ, Kim MC, Hyung WJ, Park YK, Kim W and Han SU: Efficacy of laparoscopic subtotal gastrectomy with D2 lymphadenectomy for locally advanced gastric cancer: The protocol of the KLASS-02 multicenter randomized controlled clinical trial. BMC Cancer 15: 355, 2015.

11. In H, Solsky I, Palis B, Langdon-Embry M, Ajani J and Sano T: Validation of the 8th Edition of the AJCC TNM staging system for gastric cancer using the national cancer database. Ann Surg Oncol 24: 3683-3691, 2017.

12. Mandard AM, Dalibard F, Mandard JC, Marnay J, Henry-Amar M, Petiot JF, Roussel A, Jacob JH, Segol P and Samama G: Pathologic assessment of tumor regression after preoperative chemoradiotherapy of esophageal carcinoma. Clinicopathologic correlations. Cancer 73: 2680-2686, 1994.

13. Eisenhauer EA, Therasse P, Bogaerts J, Schwartz LH, Sargent D, Ford R, Dancey J, Arbuck S, Gwyther S, Mooney M, et al: New response evaluation criteria in solid tumours: Revised RECIST guideline (version 1.1). Eur J Cancer 45: 228-247, 2009.

14. National Comprehensive Cancer Network: NCCN Clinical Practice Guidelines in Oncology Gastric Cancer (version 2.2018) National Comprehensive Cancer Network, Fort Washington, DC, 2018.

15. Lee J and Kim W: Long-term outcomes after laparoscopy-assisted gastrectomy for advanced gastric cancer: Analysis of consecutive 106 experiences. J Surg Oncol 100: 693-698, 2009.

16. DuXH,LiR,ChenL, ShenD,LiSY andGuoQ:Laparoscopy-assisted D2 radical distal gastrectomy for advanced gastric cancer: Initial experience. Chin Med J (Engl) 122: 1404-1407, 2009.

17. Yu J, Hu J, Huang C, Ying M, Peng X, Wei H, Jiang Z, Du X, Liu Z, Liu H, et al: The impact of age and comorbidity on postoperative complications in patients with advanced gastric cancer after laparoscopic D2 gastrectomy: Results from the Chinese laparoscropic gastrointestinal surgery study (CLASS) group. Eur J Surg Oncol 39: 1144-1149, 2013.

18. Hu Y, Ying M, Huang C, Wei H, Jiang Z, Peng X, Hu J, Du X, Wang B, Lin F, et al: Oncologic outcomes of laparoscopy-assisted gastrectomy for advanced gastric cancer: A large-scale multicenter retrospective cohort study from China. Surg Endosc 28: 2048-2056, 2014

19. Hu Y, Huang C, Sun Y, Su X, Cao H, Hu J, Xue Y, Suo J, Tao K, He X, et al: Morbidity and mortality of laparoscopic versus open D2 distal gastrectomy for advanced gastric cancer: A randomized controlled trial. J Clin Oncol 34: 1350-1357, 2016.

20. Li Z, Shan F, Wang Y, Li S, Jia Y, Zhang L, Yin D and Ji J: Laparoscopic versus open distal gastrectomy for locally advanced gastric cancer after neoadjuvant chemotherapy: Safety and short-term oncologic results. Surg Endosc 30: 4265-4271, 2016.

21. Kinoshita $\mathrm{T}$ and Kaito A: Current status and future perspectives of laparoscopic radical surgery for advanced gastric cancer. Transl Gastroenterol Hepatol 2: 43, 2017.

22. Li C, Oh SJ, Kim S, Hyung WJ, Yan M, Zhu ZG and Noh SH: Macroscopic Borrmann type as a simple prognostic indicator in patients with advanced gastric cancer. Oncology 77: 197-204, 2009.

23. Lauren P: The two histologic main types of gastric carcinoma: Diffuse and so-called intestinal type carcinoma, an attempt at a histo-clinical classification. Acta Parhol Microb Scan 64: 31-49, 1965 .

This work is licensed under a Creative Commons Attribution-NonCommercial-NoDerivatives 4.0 International (CC BY-NC-ND 4.0) License. 